\title{
Segmentation of 4D Cardiac MR Images Using a Probabilistic Atlas and the EM Algorithm
}

\author{
M. Lorenzo-Valdés ${ }^{1}$, G. I. Sanchez-Ortiz ${ }^{1}$, R. Mohiaddin ${ }^{2}$, and D. Rueckert ${ }^{1}$ \\ 1 Visual Information Processing Group, Department of Computing \\ Imperial College of Science, Technology, and Medicine, \\ 180 Queen's Gate, London SW7 2BZ, United Kingdom \\ 2 Royal Brompton and Harefield NHS Trust, Sydney Street \\ London, United Kingdom
}

\begin{abstract}
In this paper an automatic atlas-based segmentation algorithm for $4 \mathrm{D}$ cardiac MR images is proposed. The algorithm is based on the $4 \mathrm{D}$ extension of the expectation maximisation (EM) algorithm. The EM algorithm uses a 4D probabilistic cardiac atlas to estimate the initial model parameters and to integrate a-priori information into the classification process. The probabilistic cardiac atlas has been constructed from the manual segmentations of 3D cardiac image sequences of 14 subjects. It provides space and time-varying probability maps for the left and right ventricle, the myocardium, and background structures such as the liver, stomach, lungs and skin. In addition to the probabilistic cardiac atlas, the segmentation algorithm incorporates spatial and temporal contextual information by using 4D Markov Random Fields (MRF). Validation against manual segmentations and computation of the correlation between manual and automatic segmentation on 249 3D volumes were calculated. Results show that the procedure can successfully segment the left ventricle $(\mathrm{LV})(\mathrm{r}=0.95)$, myocardium $(\mathrm{r}=0.83)$ and right ventricle (RV) $(\mathrm{r}=0.91)$.
\end{abstract}

\section{Introduction}

In Magnetic Resonance Imaging (MRI) of the cardiovascular system, an accurate identification of the borders of the ventricles and myocardium is essential to quantitatively analyse cardiac function such as ejection fraction or wall motion thickening. Segmentation of such images needs to be automated in order to be clinically valuable and to avoid time-consuming and partly subjective manual delineation. Segmentation of cardiac images is not trivial since the images are noisy, the edges are blurred and they can have motion artifacts. Several approaches have been proposed for the automatic segmentation of cardiac structures in MR images (for a review see [1]). Recently, a number of techniques which are based on the use of a model or an atlas have been proposed 23.4. In these approaches a statistical model or atlas is used to incorporate a-priori information which enables the use of both intensity and spatial information during the segmentation process. In particular, several techniques based on active appearance models (AAM) have emerged showing improved reliability and consistency 
[5]. However, the applicability is restricted to the MR imaging sequence used for training since the intensity appearance and distribution is an explicit part of the statistical model, i.e. an active appearance model trained on spin-echo MR images is not necessarily useful for the segmentation of TrueFISP MR images of the heart. Most of these techniques work only for $2 \mathrm{D}$ even though extensions to 3D have been recently proposed [6].

This paper proposes an approach which combines the expectation maximisation (EM) algorithm [7] and a 4D probabilistic atlas of the heart for the automatic segmentation of $4 \mathrm{D}$ cardiac MR images. Methods based on the EM algorithm have been previously proposed for the classification of MR images of the brain [8]. Others authors [10]1] have proposed to include contextual information into the EM algorithm by means of Markov Random Fields (MRF). In this work we use an extension of the EM algorithm to 4D (space and time) to segment a complete 4D sequence of cardiac images. We show that introducing contextual information in $4 \mathrm{D}$ into the EM algorithm significantly improves the consistency of the segmentation process. We also use a $4 \mathrm{D}$ probabilistic cardiac atlas to include spatially and temporally varying a-priori information into the EM segmentation. The following section introduces the EM algorithm and the probabilistic atlas. Section 3 describes the automatic segmentation technique for $4 \mathrm{D}$ cardiac images. Section 4 presents some results of the proposed segmentation approach applied to 4D cardiac MR images. Finally, section 5 discusses the results and proposes future research.

\section{Methods}

\subsection{EM Algorithm}

The EM algorithm [7] is an iterative procedure that estimates the maximum likelihood for the observed data by maximising the likelihood for the estimated complete data. The complete data comprises of the observed data and the missing data. The algorithm consists of two steps: The first one is the expectation step, where the missing data are estimated by finding the maximum likelihood parameter estimates for the observed data. The second step is the maximisation step, where the maximum likelihood for the observed data are estimated by maximising the likelihood for the estimated complete data. In our case, the observed data are the signal intensities of the MR image sequence, and the missing data is the correct classification of the image sequence accomplished with help of the parameters that describe the mean and variance of each class (anatomical structure), which are usually modelled by a Gaussian distribution. Figure 1 shows an example of the different anatomical structures visible in a short-axis MR image of the heart and the corresponding intensity distribution. Given a set of $K$ classes, the probability that class $k$ has generated voxel value $y_{i}$ at position $i$ is given by the classification step: 


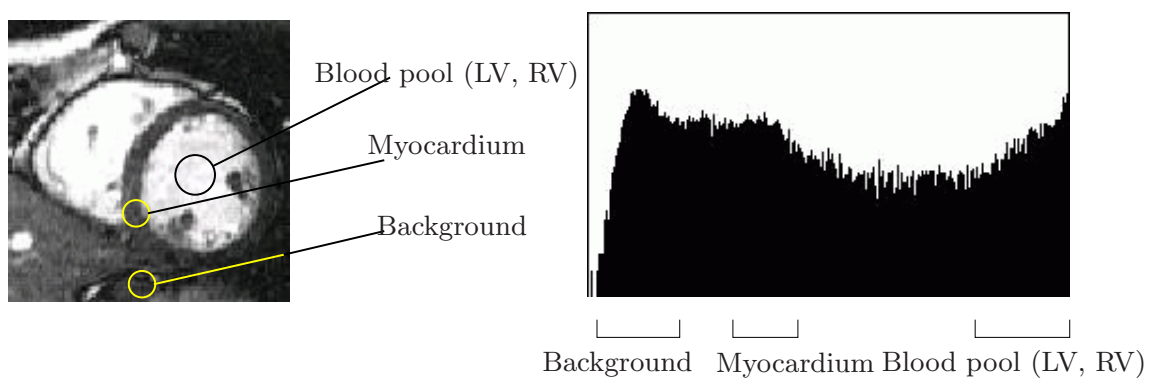

Fig. 1. A short-axis MR image of the heart showing sample regions of the blood pool of the left ventricle (LV), right ventricle (RV), myocardium, and the corresponding histogram of the intensity distribution of the image.

$$
p\left(\Gamma_{i}=k \mid y_{i}, \phi_{k}\right)=\frac{p\left(y_{i} \mid \Gamma_{i}=k, \phi_{k}\right) p\left(\Gamma_{i}=k\right)}{\sum_{j=1}^{K} p\left(y_{i} \mid \Gamma_{i}=j, \phi_{j}\right) p\left(\Gamma_{i}=j\right)}
$$

where $\Gamma_{i} \in\{k \mid k=1 \cdots K\}$ and $\phi_{k}=\left\{\mu_{k}, \sigma_{k}\right\}$. Here $\mu_{k}$ is the mean intensity of class $k$ and $\sigma_{k}^{2}$ is the variance of the intensities in class $k$. The estimation of the parameters of each class is given by:

$$
\begin{gathered}
\mu_{k}=\frac{\sum_{i} y_{i} p\left(\Gamma_{i}=k \mid y_{i}, \phi\right)}{\sum_{i} p\left(\Gamma_{i}=k \mid y_{i}, \phi\right)} \\
\sigma_{k}^{2}=\frac{\sum_{i} p\left(\Gamma_{i}=k \mid y_{i}, \phi\right)\left(y_{i}-\mu_{k}\right)^{2}}{\sum_{i} p\left(\Gamma_{i}=k \mid y_{i}, \phi\right)}
\end{gathered}
$$

The equations can be solved by iterating alternatingly between classification (Eq. 1) and parameter estimation (eqs. 2] and 3). In our implementation we are considering four distinct classes corresponding to the left ventricle (LV), myocardium, right ventricle (RV) and background.

\subsection{Markov Random Fields}

In order to improve the classification process and incorporate contextual information, Leemput et al. 10] propose the use of MRFs where other constraints are added taking into account the neighbouring voxels. They use a simple MRF that is defined on a so-called first-order neighbourhood system, i.e., only the six nearest neighbours on a $3 \mathrm{D}$ lattice are used: $\mathcal{N}_{i}=\left\{i^{n}, i^{s}, i^{w}, i^{e}, i^{t}, i^{b}\right\}$ denotes the neighbourhood of voxel $i$ where $i^{n}, i^{s}, i^{w}$ and $i^{e}$ are its four neighbours in short-axis direction, and $i^{t}, i^{b}$ its two neighbours in the long-axis direction. In addition, in our approach, we are also considering the voxels of the neighbouring time frames of the sequence. Furthermore, we assume that the spatial interaction between voxels and its neighbours is different in the temporal direction. 
For short-axis images with a typical slice thickness of $10 \mathrm{~mm}$, the contribution of neighbours in the long-axis direction is not significant when the MRF parameters are calculated, therefore we do not consider them in the calculation of the MRF parameters. Following Leemput et al. [10] we use the following Potts model to represent the spatial and temporal interactions:

$$
U_{m r f}\left(\Gamma \mid \phi_{\Gamma}\right)=\sum_{i=1}^{n} \Gamma_{i}^{T} G g_{i}+\Gamma_{i}^{T} H h_{i}
$$

Here $g_{i}=\Gamma_{i^{n}}+\Gamma_{i^{s}}+\Gamma_{i^{w}}+\Gamma_{i^{e}}$ is a vector that counts per class $k$ the number of spatial neighbours of $i$ that belong to $k$. Similarly, $h_{i}=\Gamma_{i^{t-1}}+\Gamma_{i^{t+1}}$ counts per class $k$ the number of neighbours in the temporal direction that belong to $k$. $G$ and $H$ are $K \times K$ matrices that together form the MRF parameters $\Phi_{\Gamma}=\{G, H\}$. Equations (2) and (3) remain the same but the classification step is no longer given by (1) but by

$$
p_{i k}^{(m+1)} \equiv\left[p_{i}^{(m+1)}\right] \simeq \frac{f\left(y_{i} \mid \Gamma=e_{k}, \Phi_{y}^{(m)}\right) f\left(\Gamma=e_{k} \mid p_{\mathcal{N}_{i}}^{(m)}, \Phi_{\Gamma}^{(m)}\right)}{\sum_{j}^{K} f\left(y_{i} \mid \Gamma=e_{j}, \Phi_{y}^{(m)}\right) f\left(\Gamma=e_{j} \mid p_{\mathcal{N}_{i}}^{(m)}, \Phi_{\Gamma}^{(m)}\right)}
$$

where

$$
f\left(\Gamma_{i}=e_{k} \mid p_{\mathcal{N}_{i}}^{(m)}, \Phi_{\Gamma}^{(m)}\right)=\frac{e^{-U_{m r f}\left(e_{k} \mid p_{\mathcal{N}_{i}}^{(m)}, \Phi_{\Gamma}^{(m)}\right)}}{\sum_{j=1}^{K} e^{-U_{m r f}\left(e_{j} \mid p_{\mathcal{N}_{i}}^{(m)}, \Phi_{\Gamma}^{(m)}\right)}}
$$

and $e_{k}$ is a binary vector with 1 at the $k$ th component and 0 everywhere else 1 The calculation of the MRF parameters $\Phi_{\Gamma}=\{G, H\}$ can be solved by using the least squares (LS) fit procedure described in [12]. The vector $\phi=\left[G_{11} \cdots G_{1 K}\left|G_{21} \cdots G_{2 K}\right| \cdots\left|G_{K 1} \cdots G_{K K}\right| H_{11} \cdots H_{1 K} \mid\right.$ $\left.H_{21} \cdots H_{2 K}|\cdots| H_{K 1} \cdots H_{K K}\right]^{T}$ denotes all parameters $G$ and $H$ and a vector $v_{\Gamma_{i}, g_{i}, h_{i}}=\left[\Gamma_{i 1} g_{i}^{T} \cdots \Gamma_{i K} g_{i}^{T} \mid \Gamma_{i 1} h_{i}^{T} \cdots \Gamma_{i K} h_{i}^{T}\right]^{T}$ where the following holds:

$$
\left[v_{\Gamma_{i}, g_{i}, h_{i}}^{T}-v_{\Gamma_{i}^{\prime}, g_{i}, h_{i}}^{T}\right] \phi=\log \frac{f\left(\Gamma_{i}^{\prime}, g_{i}, h_{i} \mid \Phi_{\Gamma}\right)}{f\left(\Gamma_{i}, g_{i}, h_{i} \mid \Phi_{\Gamma}\right)}
$$

The probability density function $f\left(\Gamma_{i}, g_{i}, h_{i} \mid \Phi_{\Gamma}\right)$ can be estimated using histogram techniques.

\subsection{Construction of a Probabilistic Atlas of the Heart}

The cardiac atlas has been constructed from 14 subjects and has three different components: The first component consists of spatially and temporally varying $4 \mathrm{D}$ probabilistic maps of four distinct anatomical structures of the heart (LV,

\footnotetext{
${ }^{1}$ The $\Gamma_{i}$ 's are K-dimensional vectors such that $\Gamma_{i}=e_{k}$ for some $k$.
} 


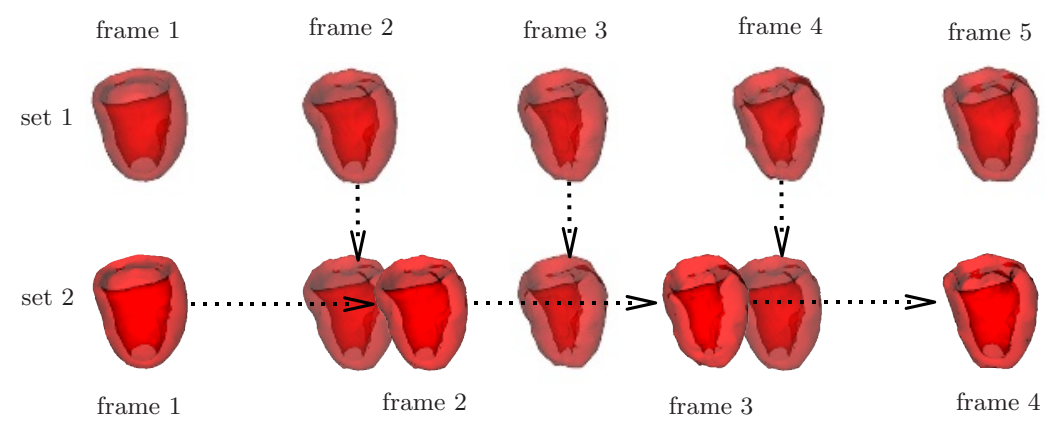

Fig. 2. Temporal registration and resampling [13]: Linear interpolation is used to generate time frames from image sequence 2 which correspond to those of sequence 1.

myocardium, RV and background). These probability maps encode prior knowledge about the cardiac anatomy and its natural spatial and temporal variability. The second component represents a $4 \mathrm{D}$ atlas of different background structures corresponding to the liver, the stomach, the left lung and the skin. The final component of the cardiac atlas corresponds to a template created by averaging the MR image sequences of all subjects used to create the atlas.

Probabilistic Maps: The purpose of the probabilistic maps is to automate the estimation of the initial parameters (mean and variance) for each class (structure) and to provide spatially and temporally varying a-priori information about the likelihood of different anatomical structures. For this purpose the $4 \mathrm{D}$ MR image sequences of 14 subjects were manually segmented. The image sequences were then resampled using shape-based interpolation 14 in order to obtain a set of images with isotropic resolution. One of the 14 subjects was randomly chosen as the reference subject and all other subjects have been aligned to this using an affine registration algorithm [15]. This spatial alignment corrects for differences due to position, size and orientation of the anatomy. In addition, we have performed a temporal alignment by matching the end-systolic and diastolic time frames of all subjects (Figure 2) using the automated algorithm described in 13. The probabilistic maps have been calculated by blurring the segmented image corresponding to each structure with a Gaussian kernel of $\sigma=8 \mathrm{~mm}$ and subsequent averaging. The final $4 \mathrm{D}$ probabilistic atlas consists of 20 time frames, and each time frame consists of a volume of $256 \times 256 \times 100$ voxels. An example of the probability maps for background, left and right ventricle and myocardium at end diastole is shown in Figure 3. The framework for the construction of the probabilistic atlas is illustrated in Figure 4 .

Background Map: In addition to the probabilistic maps representing the structures of interest, the background was divided into 4 subclasses corresponding to the liver, the stomach, the left lung and the skin as shown in Figure [5(a). For this purpose the background structures of the reference subject were man- 
ually segmented and resampled using shape-based interpolation. This 4D background map was used to estimate the model parameters (mean and variance) of the different structures in the background. The reason why this background map is needed is that the background contains various organs and modelling it using a single Gaussian would not be sufficient since it contains a wide range of intensities. These regions were considered as subclasses since they were only used to estimate the parameters of the background. They were not used for the MRF calculation or for the probabilistic atlas since they were not structures of interest in the segmentation.

Template: In addition, we have also constructed a 3D template of the heart during end diastole. This 3D template (Figure 5(b)) has been calculated by normalising and averaging the intensities of all end-diastolic images, after spatial alignment to the reference image (Figure[5(c)). The purpose of this 3D template is to allow us to align the cardiac atlas with the images to be segmented.

\section{Automatic Segmentation}

In the first step of the automatic segmentation the 3D template was registered to the end-diastolic time frame of the MR sequence by using an affine registration. This produces a transformation which spatially aligns the $4 \mathrm{D}$ probabilistic atlas to the MR image sequence. A temporal alignment was performed as described in Figure 2. Subsequently, a mask was generated for each class (LV, $\mathrm{RV}$, myocardium and background) in the probabilistic atlas by using only those areas that had a probability higher than $50 \%$ of belonging to that class. For the background each subclass was used as a mask. Since the atlas was already aligned with the MR image, each of these masks was used to calculate the initial model parameters (mean and variance) of each class and subclass. Having the initial model parameters allows us to perform the first classification of the image sequence by assigning the class with the highest probability for a voxel at position $i$. The background had four probabilities corresponding to each subclass

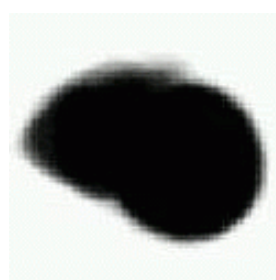

(a)

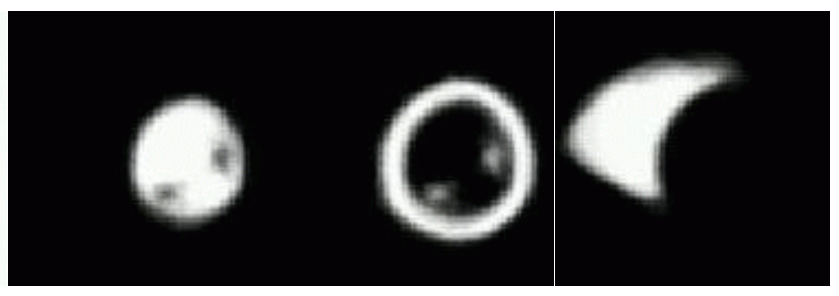

(c) (d)

Fig. 3. A probabilistic atlas of the heart constructed from 14 subjects: (a), (b), (c) and (d) correspond to probability maps for background, LV, myocardium and RV, shown at end diastole (note that the atlas is $4 \mathrm{D}$, i.e. the probability maps change over time). Dark areas indicate low probability, bright areas indicate high probability. 


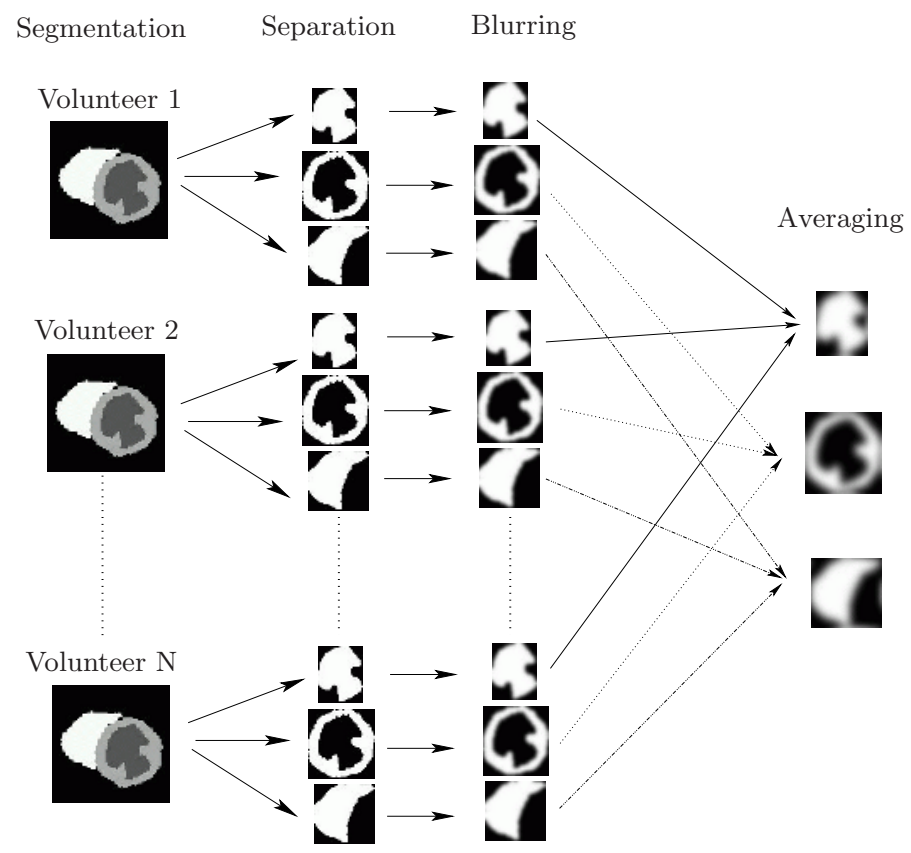

Fig. 4. Construction of the probabilistic atlas.

and only the highest was considered as the probability of the background of that specific voxel. In summary, the EM algorithm interleaves four steps: classification of the voxels with the initial parameters (Eq. 1), estimation of the Gaussian parameters (eqs. 2] and 3), estimation of the MRF parameters (Eq. (7) and classification using all the parameters (Eq. 51). It is important to note that the probabilistic atlas constrains the classification since it provides a spatially and temporally varying prior probability for each tissue class $k$. Classification using the EM algorithm was repeated until the values of the parameters did not change significantly. All the parameters (mean, variance and MRF model) were re-estimated at each iteration.

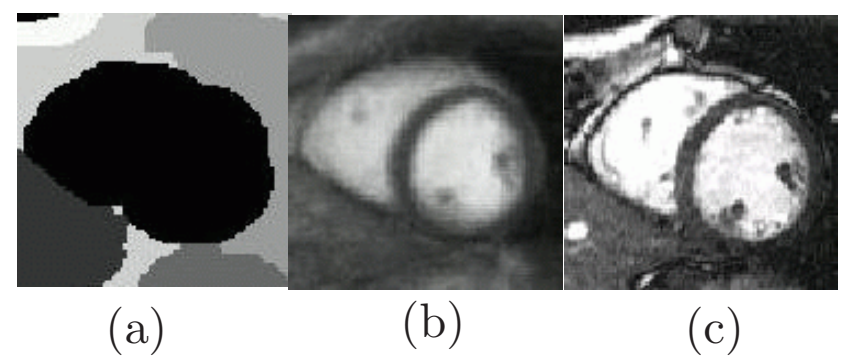

Fig. 5. Image (a) shows the subclasses of the background, image (b) is the template used to align the atlas with the $4 \mathrm{D}$ MR images and (c) is the image used as the reference. 

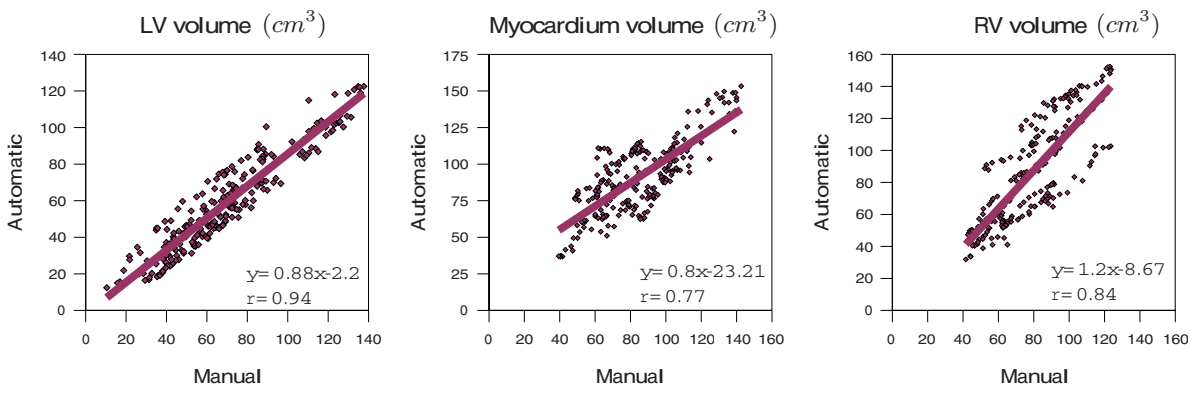

Fig. 6. Comparison of the volumes of 249 3D images obtained by manual and automatic segmentation considering neighbours in the short-axis direction only and using the probabilistic atlas.

\section{Results}

Cardiac short-axis images were acquired at Royal Brompton Hospital, London, UK, from 12 healthy volunteers using a Siemens Sonata $1.5 \mathrm{~T}$ scanner with a TrueFisp sequence and $256 \times 256 \times 10$ voxels. Similarly, two more image sequences were acquired at Guy's Hospital, London, UK using a Philips Gyroscan Intera 1.5T scanner. Each image sequence consisted of 10 to 26 time frames, involving a total of 249 volumetric datasets. The field of view ranged between 300-350 mm, the thickness of slices was $10 \mathrm{~mm}$ and the total acquisition time was approximately 15 minutes. In order to avoid bias, we used the 'leave one out' test where the image set to be segmented was not used in the construction of its corresponding atlas. The results of the automatic segmentation were compared against those obtained by manually segmenting the $144 \mathrm{D}$ image sequences. In order to assess the performance of the automatic segmentation the volumes of the ventricles and myocardium were calculated and linear regression analysis was used to compare the manually and the automatically segmented images. Figure 6 shows the results for the automatic segmentation when considering only the neighbouring voxels in the short-axis directions. The correlation for the $\mathrm{LV}$, myocardium and RV is $r=0.94, r=0.77$ and $r=0.84$, respectively. Figure 7 presents the results of the automatic segmentation including also the neighbouring voxels in the temporal direction where an improvement is noticeable, especially in the myocardium $(r=0.83)$ and RV $(r=0.91)$. The results for the LV are largely unchanged $(r=0.95)$. An example of segmentation is shown if Fig 8 . The automatic segmentation for a complete sequence of 26 frames took 25 minutes with an average of 20 iterations for the EM algorithm to converge on a PC platform with a 2 Ghz processor.

\section{Discussion and Future Work}

We have presented a method for the automatic segmentation of $4 \mathrm{D}$ cardiac $\mathrm{MR}$ images. We have demonstrated that the combination of the EM algorithm and 

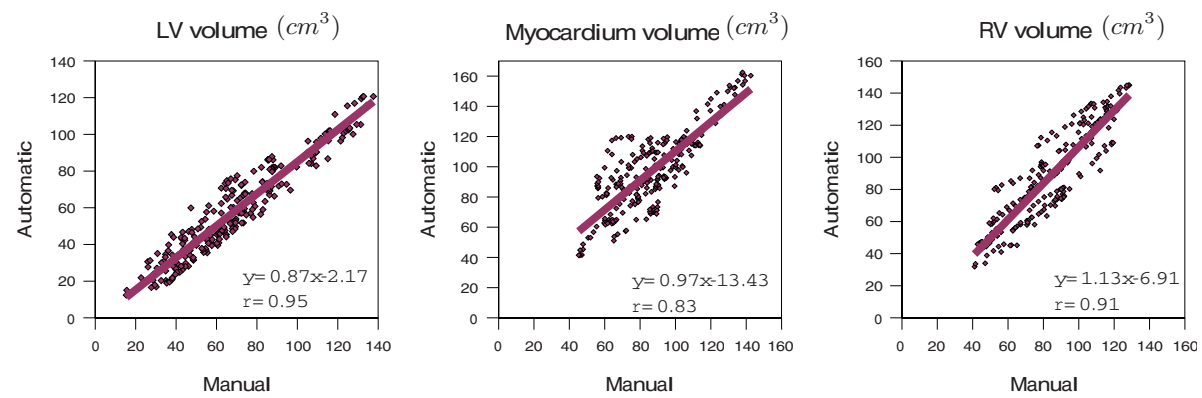

Fig. 7. Comparison of the volumes of 249 3D images obtained by manual and automatic segmentation considering neighbours in the spatial and temporal directions and using the probabilistic atlas.

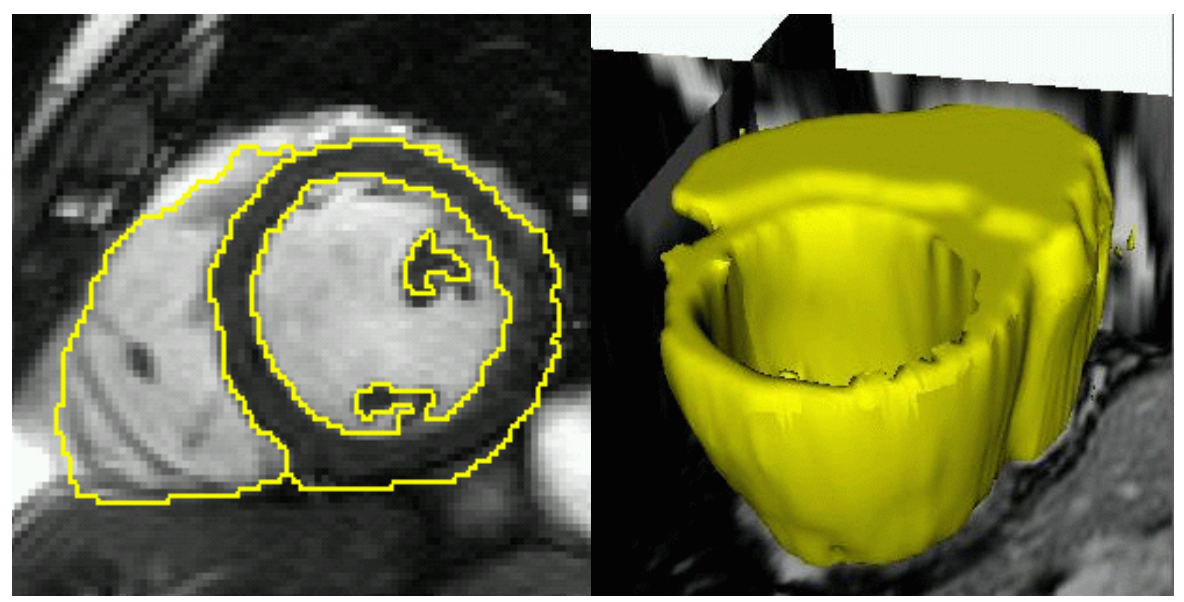

Fig. 8. Example of typical segmentation results overlaid on 2D and 3D images.

a probabilistic atlas yields an accurate and fast segmentation of a complete cardiac sequence. Using a probabilistic atlas as a spatially and temporally varying prior was essential for segmenting structures with a similar range of intensities such as the LV and RV or the myocardium and some structures in the background. Since the probabilistic maps represent a 4D atlas, a good segmentation was obtained for the entire sequence despite the considerable change in the size of the structures during the cardiac cycle. Also, using the spatio-temporal MRF approach for the EM algorithm improved the results significantly compared to spatial MRFs only. Our approach is completely automatic and consistent since the alignment of the atlas is performed automatically by an affine registration algorithm. In addition, it is independent of the image intensities in the MR images and can be applied to images acquired with other sequences (e.g. black-blood studies) since the estimation of the parameters is performed using the atlas. The 
major limitation is that there could be misclassified voxels at the boundaries of the classes and that those can be slightly overestimated, especially when vessels are very close to the ventricles or when the contrast between myocardium and surrounding tissues is very low. This can also happen when the difference between neighbouring time frames is too big. Future work will include other cardiac structures such as the left and right atria and some of the main arteries and vessels to prevent them from being classified as part of the ventricles.

Acknowledgements. M. Lorenzo-Valdés is funded by a grant from CONACyT, México. G. I. Sanchez-Ortiz is funded by EPSRC grant no. GR/R41002/01.

\section{References}

1. J. S. Suri. Computer vision, pattern recognition and image processing in left ventricle segmentation: The last 50 years. Pattern Analysis and Applications, 3(3): 209-242, 2002.

2. F. Vincent, P. Clarysse, P. Croiselle, and I. Magnin. Segmentation of the heart from MR image sequences using a 3D active model. Annals of Biomedical Engineering, 28(1): S-42, 2000.

3. B.P.F. Lelieveldt, R.J. Van der Geest, R.M. Ramze, J.G. Bosch, and J.H.C. Reiber. Anatomical model matching with fuzzy implicit surfaces for segmentation of thoracic volume scans. IEEE Transactions on Medical Imaging, 18(3): 218-230, 1999.

4. M. Lorenzo-Valdes, G. I. Sanchez-Ortiz, R. Mohiaddin, and D. Rueckert. Atlasbased segmentation and tracking of $3 \mathrm{D}$ cardiac MR images using non-rigid registration. In Fifth Int. Conf. on Medical Image Computing and Computer-Assisted Intervention (MICCAI '02), Lecture Notes in Computer Science, pages 642-650. Springer-Verlag, 2002.

5. S.C. Mitchell, B.P.F. Lelieveldt, R.J. Van Der Geest, H. G. Bosch, J.H.C. Reiber, and M. Sonka. Multistage hybrid active appearance model matching: Segmentation of left and right ventricles in cardiac MR images. IEEE Transactions on Medical Imaging, 20: 415-423, 2001.

6. S.C. Mitchell, B.P.F. Lelieveldt, J. G. Bosch, R. Van Der Geest, J.H.C. Reiber, and M. Sonka. Segmentation of cardiac MR volume data using 3D active appearance models. In SPIE Conference on Medical Imaging, Image Processing, pages 433-443, 2002.

7. A. P. Dempster, N. M. Laird, and D. B.Rubin. Maximum likelihood from incomplete data via the EM algorithm. Journal Royal Statistical Society A, 39: 1-38, 1977.

8. W. M. Wells III, W. E. L. Grimson, R. Kikinis, and F. A. Jolesz. Adaptive segmentation of MRI data. IEEE Transactions on Medical Imaging, 15(4): 429-442, 1996.

9. Z. Liang, J. R. MacFall, and D. P. Harrington. Parameter estimation and tissue segmentation from multispectral MR images. IEEE Transactions on Medical Imaging, 13(3): 441-449, 1994.

10. K. Van Leemput, F. Maes, D. Vandermeulen, and P. Suetens. Automated modelbased tissue classification of MR images of the brain. IEEE Transactions on Medical Imaging, 18(10): 897-908, 1999. 
11. Y. Zhang, M. Brady, and S. Smith. Segmentation of brain MR images through a hidden markov random field model and the expectation-maximization algorithm. IEEE Transactions on Medical Imaging, 20: 45-57, 2001.

12. S. Z. Li. Markov Random Field Modeling in Computer Vision. Springer-Verlag, Berlin, Germany, 1995.

13. D. Perperidis, A. Rao, M. Lorenzo-Valdes, R. Mohiaddin, and D. Rueckert. Spatiotemporal alignment of $4 \mathrm{D}$ cardiac MR images. In FIMH Conference on Functional Imaging and Modeling of the Heart, 2003. In press.

14. S.P. Raya and J.K. Udipa. Shape-based interpolation of multidimensional objects. IEEE Transactions on Medical Imaging, 9(1): 32-42, 1990.

15. C. Studholme, D.L.G. Hill, and D.J. Hawkes. Automated three-dimensional regisration of magnetic resonance and positron emission tomography brain images by multiresolution optimization of voxel similarity measures. Medical Physics, 24(1): 71-86, 1997. 